\title{
Research Paper: Clinical Manifestations of Mania in Patients With Bipolar I Disorder Based on the Primary crossuark Symptoms in DSM-5
}

\author{
Zahra Mohammadi ${ }^{1}$, Abbas Pourshahbaz ${ }^{1 *}$, Behrooz Dolatshahi', Marjan Poshtmashhadi ${ }^{1}$
}

1. Department of Clinical Psychology, University of Social Welfare and Rehabilitation Sciences, Tehran, Iran.

Cltation: Mohammadi, Z., Pourshahbaz, A., Dolatshahi, B., \& Poshtmashhadi, M. (2017). Clinical Manifestations of Mania in Patients With Bipolar I Disorder Based on the Primary Symptoms in DSM-5. Journal of Practice in Clinical Psychology, 5(4), $289-296$. https://doi.org/10.29252/NIRP.JPCP.5.4.289

: https://doi.org/10.29252/NIRP.JPCP.5.4.289

\section{Article info:}

Received: 30 Mar. 2017

Accepted: 13 Jul. 2017

\section{Keywords:}

Bipolar disorder, Mania, Clinical manifestations

\section{ABSTRACT}

Objective: According to DSM-5, bipolar disorder is a condition in which the patient experiences one or several manic episodes and sometimes major depressive episodes too. The signs and symptoms of the disorders in DSM are generally influenced by cultural and ethnic factors. Therefore, the present study was aimed at identifying the clinical manifestations of mania in bipolar I disorder in Iranian population.

Methods: The present work is a cross-sectional study. The participants included 64 patients in the manic phase of bipolar I disorder who were selected from the Razi psychiatric hospital and the Taleghani Hospital, using a convenience sampling method. The study data were gathered using the Structured Clinical Interview for DSM-IV Axis I Disorders (SCID-I) and the Young Mania Rating Scale (YMRS).

Results: According to the findings, the most common signs and symptoms of mania in hospitalized patients with bipolar I disorder included reduced need for sleep (89.1), poor insight (87.5), elevated mood (85.9), talkativeness (79.7), and psychotic features (68.8). The major symptoms of the patients according to DSM-5 criteria included reduced need for sleep (89.1), talkativeness (79.7), and psychomotor agitation (40.6). According to t-test analysis, patients with and without a history of mental disorders did not have significantly different mania scores. The one-way analysis of variance (ANOVA) analysis indicated no significant difference in mania scores based on the age of onset of the disorder, marital status (single, married, divorced, or widow), education level, and number of hospitalizations. The chi-squared test revealed that patients with a lower education level showed more aggressive behaviors.

Conclusion: According to our results, since the response of a patient to a particular treatment is largely influenced by the symptoms of the disorder, clinicians should pay critical attention to the most common signs and symptoms of any mania.

\footnotetext{
* Corresponding Author:

Abbas Pourshahbaz, PhD

Address: Department of Clinical Psychology, University of Social Welfare and Rehabilitation Sciences, Tehran, Iran.

Tel: +98 (939) 3391589

E-mail: apourshahbaz@yahoo.com
} 


\section{Introduction}

ccording to Diagnostic and Statisti1 cal Manual of Mental Disorders, Fifth Edition (DSM-5), bipolar disorder is a condition in which the patient experiences one or several manic episodes, and sometimes also major depressive episodes (Whitbourne \& Halgin, 2014).

The prevalence of bipolar I disorder is estimated to be between 0 and 0.6 percent (American Psychiatric Association, 2013). This condition leads to a significant decrease in job performance, which in turn results in a lower socioeconomic status for the patients, despite having a similar level of education to that of their peers in the general population (American Psychiatric Association, 2013).

Consequently, bipolar I disorder reduces the quality of life of the patients and imposes different costs on them, their families, and the society (Van der Voort et al., 2015; Sammarco, 2016). Mania is the major clinical manifestation of bipolar I disorder (Proudfoot, Doran, Manicavasagar, \& Parker, 2011). In fact, patients in the manic phase are diagnosed with bipolar I disorder, even when there are no depression episodes (American Psychiatric Association, 2013). Patients' experiences during mania are very complex (Goodwin \& Jamison, 2007). They may seem extremely sociable, talkative, vigilant, witty, and over-confident. Their openness and high energy can lead to serious dysfunctions. They may have a severe false self-esteem and grandiose or even psychotic thinking. In addition, they may experience racing thoughts, become easily distracted, and need excessive stimulation. Manic patients are very energetic. However, euphoria can suddenly change into severe irritability or even aggression or hostility, especially if other people try to stop their unreasonable or grandiose plans (American Psychiatric Association, 2013).

Although DSM-5 includes diagnostic criteria for bipolar I disorder, it must be noted that the severity and manifestations of bipolar symptoms are determined by multiple factors, including environmental and biological ones (Lieberman, Massey, \& Goodwin, 2010). Bipolar I disorder is equally common in men and women, but men tend to have significantly more manic episodes as compared to women (Sadock \& Sadock, 2011); however, the age of onset of mania is higher in women, and two peak ages are particularly seen in women viz., early adulthood and midlife (Kroon et al., 2013).

Patients having bipolar disorder with an earlier age of onset suffer from more psychotic symptoms than those with an older age of onset, and this makes the clinical manifestations of their disorder even more severe (Carlson, Bromet, \& Sievers, 2000; Schulze et al., 2002). In addition, since their condition has started at an early age, these patients usually do not have much educational qualification. Bipolar I disorder is more common in single, divorced, or widowed individuals compared to married people (Sadock \& Sadock, 2011). Given the differences in demographic variables and other cultural factors, the most common symptoms of mania in bipolar I disorder in the US include increased psychomotor activity, racing thoughts, pressure of talking, and reduced need for sleep (Cassidy \& Carroll, 2001), while in the UK, it includes talkativeness, psychomotor agitation, and reduced need for sleep (Goodwin \& Jamison, 2007).

The socio-cultural issues in each society affect the clinical presentations of mental disorders; however, these issues have not received enough attention in the universal classifications like DSM. Given that the symptoms of manic episodes in bipolar I disorder are culture-bound, knowing their frequency in each society can lead to a more accurate diagnosis of bipolar I disorder. Therefore, the goal of the present study is to examine the clinical manifestation of a manic episode in Iranian patients with bipolar I disorder.

\section{Methods}

The present research work is a descriptive cross-sectional study. The statistical population included all patients with bipolar I disorder in the Razi psychiatric hospital and the Taleghani Hospital in 2016-2017. Among this population, a total of 97 patients with bipolar I disorder were selected using a convenience sampling method, and were examined in terms of the inclusion and exclusion criteria; 64 patients, who met the criteria, were included in the analysis. The inclusion criteria were as follows: diagnosed with bipolar disorder according to SCID-I, aged between 18 to 65 years, and being able to understand the study questions. The exclusion criteria included severe mental retardation, severe physical disorders, and the unwillingness of patients to participate in the study. The following instruments were used to collect data:

The Structured Clinical Interview for DSM-IV Axis I Disorders (SCID-I): This is a semi-structured interview used to diagnose the major disorders of axis I, according to DSM-IV criteria. SCID-I has two versions: A research version (SCID-I-RV) and a clinical version (SCID-CV). The research version includes the axis I disorders with complete details and is very useful in studies that require accurate details, whereas the clinical version only 
includes disorders commonly observed in clinics. Testretest reliability estimates of 0.40 (dysthymic disorder) and 0.84 (bipolar disorder) have been reported for the SCID-I (Mohammadkhani, Jahani Tabesh, \& Tamanaiefar, 2013). In the present study, the SCID-CV was used. Demographic questionnaire: A researcher-made demographic questionnaire was used to gather data on gender, age, educational level, marital status, age of onset of the disorder, family history of mental disorders, and number of hospitalizations, if any.

The Young Mania Rating Scale (YMRS): The YMRS was developed by Young, Biggs, Ziegler and Meyer (1978) based on the descriptions provided for the main symptoms of mania. It is aimed at assessing the severity of mania symptoms in patients with bipolar disorder, based on patients' self-reports of their clinical condition and clinicians' observations during clinical interviews. Reliability of the scale has been reported to range from 0.41 (appearance) to 0.85 (thought and language disorders), an inter-rater reliability of 0.93 has been reported for the total scale, and inter-rater reliability estimates ranging from 0.66 (aggressive-destructive behavior) to 0.95 (sleep) have been reported for its subscales (Young et al., 1978). In Iran, Cronbach's alphas of 0.72 and 0.63 , respectively in patient and clinical groups, and an inter-rater reliability of 0.96 have been reported for the YMRS (Barekatain, Tavakoli, Molavi, Maroufi, \& Salehi, 2007). In the present study, the reliability of the YMRS was found to be 0.72 .

Firstly, patients diagnosed with bipolar I disorder by a psychiatrist were selected. Consequently, medical records were reviewed to examine the inclusion and exclusion criteria. Then, the SCID-I was conducted to confirm the diagnosis of mania in the patients. Subsequently, demographic data were collected based on the information provided in medical records and also by interrogating the patients. Finally, the YMRS was administered to examine the clinical manifestations of manic episodes. Before completing the questionnaires, participants were informed about the study objectives and were reassured that their personal information remained confidential. In addition, participation in the study was completely voluntary, and participants were given the freedom to leave the study at any time. The study data were analyzed using independent samples t-test and one-way analysis of variance (ANOVA). Analyses were performed using the SPSS software v.22.

\section{Results}

Among the participants, $90.6 \%$ were women with a mean age of $38.22(\mathrm{SD}=11.14)$, and $9.4 \%$ were men with a mean age of $47.50(\mathrm{SD}=13.57) .39 .1 \%$ of the participants were illiterate or had primary school education, $15.6 \%$ middle school education, $14.1 \%$ high school education, and $31 \%$ a high school diploma or college education. $39.1 \%$ of the participants were single, $37.5 \%$ married, and $23.4 \%$ divorced or widowed. $60.9 \%$ of the participants had a family history of mental disorders. In $23.4 \%$ of the participants, the first symptoms had emerged in the age range of $10-20$ years, in $64.1 \%$ in the age range of $20-40$ years, and in $12.5 \%$ in the age range of $40-60$ years. $23.4 \%$ of the participants had been hospitalized only once, $21.9 \%$ twice, and the rest had three or more, earlier hospitalization records. In overall, 76.6\% had more than one hospitalization records (Table 1).

According to the data from the YMRS, the most common signs and symptoms of mania in the patients with bipolar I disorder included reduced need for sleep (89.1), poor insight (87.5), elevated mood (85.9), talkativeness (79.7), and psychotic features (68.8). In addition, the main symptoms of mania according to DSM-5 criteria included reduced need for sleep (89.1), talkativeness (79.7), and psychomotor agitation (40.6) (Table 2). Among the mania symptoms, the categories of poor insight (1.89) and aggressive- destructive behaviors (0.20) had the highest and lowest severity levels, respectively; whereas, among the main symptoms of mania based on DSM-5 criteria, reduced need for sleep (2.39) and racing thoughts $(0.25)$ had the highest and lowest severity levels, respectively. According to the results of the chi-squared test, there was no significant difference in clinical manifestations of mania based on demographic characteristics; the only exception was that those with a lower education level showed more aggressive behaviors $($ Sig. $=0.04)$.

According to the results of the independent samples t-test, there was no significant difference in mania scores between patients with or without a family history of mental disorders (Sig. $=0.51$ ). In addition, the results of ANOVA indicated no significant difference in mania scores based on the age of onset of the disorder (Sig.=0.94), marital status (Sig. $=0.63)$, education level $($ Sig. $=0.32)$, and number of hospitalizations $($ Sig. $=0.88)$ (Tables 3 and 4).

\section{Discussion}

The goal of the present study was to identify the clinical manifestations of mania in patients with bipolar I disorder, and to examine the differences in clinical manifestations based on demographic characteristics. In accordance with several previous reports, particularly Ken- 
Table 1. Frequency distribution of demographic variables

\begin{tabular}{|c|c|c|c|}
\hline & & Frequency & Frequency (\%) \\
\hline \multirow{2}{*}{ Gender } & Female & 58 & 90.6 \\
\hline & Male & 6 & 9.4 \\
\hline \multirow{3}{*}{ Marital status } & Single & 25 & 39.1 \\
\hline & Married & 24 & 37.5 \\
\hline & Divorced and widowed & 15 & 23.4 \\
\hline \multirow{4}{*}{ Education level } & Illiterate or primary school education & 25 & 39.1 \\
\hline & Middle school education & 10 & 15.6 \\
\hline & High school education & 9 & 14.1 \\
\hline & High school diploma or college education & 20 & 31.3 \\
\hline \multirow{2}{*}{$\begin{array}{l}\text { Family history of mental } \\
\text { disorders }\end{array}$} & Having & 39 & 60.9 \\
\hline & Not having & 25 & 39.1 \\
\hline \multirow{3}{*}{ Age of onset of the disorder } & $10-20$ & 15 & 23.4 \\
\hline & $20-40$ & 41 & 64.1 \\
\hline & $40-60$ & 8 & 12.5 \\
\hline \multirow{3}{*}{ Number of hospitalizations } & Once & 15 & 23.4 \\
\hline & Twice & 14 & 21.9 \\
\hline & Three times or more & 35 & 54.7 \\
\hline
\end{tabular}

Table 2. The most common signs and symptoms of mania according to the YMRS

\begin{tabular}{|c|c|c|c|c|}
\hline \multirow{2}{*}{ Signs and Symptoms } & \multirow{2}{*}{ Frequency } & \multirow{2}{*}{ Frequency (\%) } & \multicolumn{2}{|c|}{ Severity of Signs and Symptoms } \\
\hline & & & Mean & SD \\
\hline Reduced need for sleep* & 57 & 89.1 & 2.39 & 1.03 \\
\hline Poor insight & 56 & 87.5 & 2.84 & 1.25 \\
\hline Elevated mood & 55 & 85.9 & 1.73 & 0.97 \\
\hline Talkativeness* & 51 & 79.7 & 1.81 & 1.27 \\
\hline Psychotic features & 44 & 68.8 & 0.68 & 0.46 \\
\hline Appearance & 26 & 40.6 & 0.53 & 0.79 \\
\hline Psychomotor agitation* & 26 & 40.6 & 0.98 & 1.29 \\
\hline Distraction* & 25 & 39.1 & 0.39 & 0.49 \\
\hline Sexual desire* & 24 & 37.5 & 0.57 & 0.86 \\
\hline Megalomania* & 17 & 26.6 & 0.26 & 0.44 \\
\hline Racing thoughts* & 16 & 25.0 & 0.25 & 0.43 \\
\hline Irritability & 16 & 25.0 & 0.40 & 0.77 \\
\hline Aggressive-destructive behaviors & 10 & 15.6 & 0.20 & 0.53 \\
\hline
\end{tabular}


Table 3. Results of $t$-test for mania scores based on family history of mental disorders

\begin{tabular}{ccccc}
\hline & Mean & SD & df & P \\
\hline With family history of mental disorders & 17.51 & 3.64 & 62 & 0.51 \\
Without family history of mental disorders & 16.88 & 3.94 & & \\
\hline
\end{tabular}

Table 4. Results of ANOVA for mania scores according to demographic variables

\begin{tabular}{|c|c|c|c|c|}
\hline Variables & Mean & SD & $\mathbf{F}$ & $\mathbf{P}$ \\
\hline Age of onset of the disorder & 1.89 & 0.59 & 0.05 & 0.94 \\
\hline Marital status & 1.84 & 0.78 & 0.45 & 0.63 \\
\hline Education level & 2.37 & 1.29 & 1.18 & 0.32 \\
\hline Number of hospitalizations & 3.31 & 0.83 & 0.12 & 0.88 \\
\hline
\end{tabular}

nedy et al. (2005), Schwartzman et al. (2007), and Eid et al. (2013), we observed that despite the higher rate of manic episodes in men, more women had attended the hospitals to seek treatment and this probably could be attributed to the fact that in the manic phase, women are significantly more likely than men to be hospitalized. The study is also in line with the findings of Coryell, Fiedorowicz, Leon, Endicott and Keller (2013), indicating that the onset of mania commonly occurs at a young age.

Keeping in view of the above findings, we infer that those with a family history of mental disorders (In the present study, it was found that $60.9 \%$ of the patients with bipolar I disorder had a family history of mental disorders) have experienced more manic and depressive episodes in the past (In the present study, it was found that $76.6 \%$ of the patients had multiple hospitalizations in the past), and have a younger age of onset that is related to the genetic prediction hypothesis. Based on this hypothesis, the severity of mental disorder is increased, and the age of onset of mental disorder is decreased across consecutive generations (Grabe, 2017).

In the present study, we observed that majority of the patients with bipolar I disorder were single or divorced; this finding is consistent with those of $\mathrm{Hu}$ et al. (2017) and Gignac, McGirr, Lam and Yatham (2015). The reason why mania is more common in single or divorced patients is that those with bipolar I disorder are less likely to get married, and those who are married have dysfunctional marriages with a high possibility of extramarital affairs; therefore, they have a higher divorce rate (Sadock \& Sadock, 2015). In addition, $68.8 \%$ of the patients in this study had an education qualifications below high-school diploma, a finding in line with those of Maede et al. (2011) and Olfson et al. (2016); this could be related to the onset of mania early in life, or unwillingness of patients with a high education level to seek treatment due to social stigmas.

In the present study, the signs and symptoms of mania in the hospitalized patients with bipolar disorder included reduced need for sleep, poor insight, elevated mood, talkativeness, and psychotic features, but based on DSM5 criteria, the main symptoms of mania included reduced need for sleep, talkativeness, and psychomotor agitation; however, megalomania, reduced need for sleep, and talkativeness were particularly the most common symptoms in DSM-5. This can be attributed to different cultural backgrounds affecting the form and content of the symptoms of mental disorders. In a previous study by Akiskal et al. (1998) in France, megalomania, reduced need for sleep, talkativeness, and racing thoughts; and in a study by Angst et al. (2011), talkativeness, reduced need for sleep, and an increase in goal-directed behaviors, were respectively the most common symptoms of mania.

Largely, these findings are consistent with the present study results. Furthermore, according to this study, we infer that although euphoria and elevated mood are among the main characteristics of mania, it is not in fact a euphoric condition. In overall, in contrast to a previous report by Yazla, İnanç, and Bilici (2012), no significant difference was found between clinical manifestations of 
mania based on demographic characteristics, except that those with a lower education showed more aggressive behaviors. This could be attributed to physiological and cultural factors. In other words, patients with a lower socio-economic status may have had fewer opportunities to learn proper coping strategies to cope with stressful events. No significant difference was observed in mania scores based on age of onset of the disorder, education level, family history of mental disorders, and number of hospitalizations. This is in agreement with the findings of McElroy et al. (1995), Goi, Mosqueiro, and Cunha (2009), and Antypa and Serretti (2014).

Conclusively, the study results indicated that, based on DSM-5 criteria, reduced need for sleep, talkativeness, and psychomotor agitation were the most common mania symptoms in patients with bipolar I disorder. In addition, no significant difference was found between clinical manifestations of mania in demographic characteristics, but demographic characteristics could be considered as potential risk factors influencing the course and prognosis of the disorder. Despite the aforementioned important observations of the study, there were few limitations, such as small sample size, sample confined to hospitalized patients, and a limited number of male patients were recruited. Therefore, further studies are suggested to be conducted to work with outpatients, use a larger sample size, and inclusion of relatively more male patients in the samples.

\section{Acknowledgments}

This paper is an outcome of the first author's Master's thesis work on clinical psychology from the University of Social Welfare and Rehabilitation Sciences.

\section{Conflict of Interest}

All authors certify that this manuscript has neither been published in whole nor in part nor being considered for publication elsewhere. The authors have no conflicts of interest to declare.

\section{References}

Akiskal, H. S., Hantouche, E. G., Bourgeois, M. L., Azorin, J. M., Sechter, D., Allilaire, J.F., et al. (1998). Gender, temperament, and the clinical picture in dysphoric mixed mania: Findings from a French national study (EPIMAN). Journal of Affective Disorders, 50(2), 175-186. doi: 10.1016/s0165-0327(98)00113-x

Angst, J., Azorin, J. M., Bowden, C. L., Perugi, G., Vieta, E. Gamma, A.,et al. (2011). Prevalence and characteristics of undiagnosed bipolar disorders in patients with a major depres- sive episode: the BRIDGE study. Archives of General Psychiatry, 68(8), 791-799. doi: 10.1001/archgenpsychiatry.2011.87

Antypa, N., \& Serretti, A. (2014). Family history of a mood disorder indicates a more severe bipolar disorder. Journal of Affective Disorders, 156, 178-186. doi: 10.1016/j.jad.2013.12.013

American Psychiatric Association. (2013). Diagnostic and statistical manual of mental disorders (DSM-5®). Missouri: American Psychiatric Pub.

Barekatain, M., Tavakoli, M., Molavi, H., Maroufi, M., \& Salehi, M. (2007). Standardization, reliability and validity of the Young Mania Rating Scale. Psychology, 11(2), 150-166.

Carlson, G. A., Bromet, E. J., \& Sievers, S. (2000). Phenomenology and outcome of subjects with early-and adult-onset psychotic mania. American Journal of Psychiatry, 157(2), 213-219. doi: 10.1176/appi.ajp.157.2.213

Cassidy, F., \& Carroll, B. J. (2001). Frequencies of signs and symptoms in mixed and pure episodes of mania: implications for the study of manic episodes. Progress in Neuro-Psychopharmacology and Biological Psychiatry, 25(3), 659-665. doi: 10.1016/ s0278-5846(00)00174-3

Coryell, W., Fiedorowicz, J., Leon, A. C., Endicott, J., \& Keller, M. B. (2013). Age of onset and the prospectively observed course of illness in bipolar disorder. Journal of Affective Disorders, 146(1), 34-38. doi: 10.1016/j.jad.2012.08.031

Eid, L., Heim, K., Doucette, S., McCloskey, S., Duffy, A., \& Grof, P. (2013). Bipolar disorder and socioeconomic status: what is the nature of this relationship? International Journal of Bipolar Disorders, 1(1), 9. doi: 10.1186/2194-7511-1-9

Gignac, A., McGirr, A., Lam, R. W., \& Yatham, L. N. (2015) Course and outcome following a first episode of mania: Fouryear prospective data from the Systematic Treatment Optimization Program (STOP-EM). Journal of Affective Disorders, 175, 411-417. doi: 10.1016/j.jad.2015.01.032

Goi, P. D., Mosqueiro, B. P., \& Cunha, A. B. D. (2009). The impact of marital status in hospitalized patients with bipolar disorder. Revista Brasileira de Psiquiatria, 31(4), 394-395. doi: 10.1590/s1516-44462009000400023

Goodwin, F. K., \& Jamison, K. R. (2007). Manic-depressive illness: Bipolar disorders and recurrent depression (Vol. 1). Oxford: Oxford University Press.

Grabe, H. J. (2017). Environment, genes, and mental health anticipation and medicine. Berlin: Springer.

Hu, C., Torres, I. J., Qian, H., Wong, H., Halli, P., Dhanoa, T., et al. (2017). Trajectories of body mass index change in first episode of mania: 3-year data from the Systematic Treatment Optimization Program for Early Mania (STOP-EM). Journal of Affective Disorders, 208(15), 291-297. doi: 10.1016/j. jad.2016.08.048

Kennedy, N., Boydell, J., Kalidindi, S., Fearon, P., Jones, P. B. Van Os, J., et al. (2005). Gender differences in incidence and age at onset of mania and bipolar disorder over a 35-year period in Camberwell, England. American Journal of Psychiatry, 162(2), 257-262. doi: /10.1176/appi.ajp.162.2.257

Kroon, J. S., Wohlfarth, T. D., Dieleman, J., Sutterland, A. L., Storosum, J. G., Denys, D., et al. (2013). Incidence rates and risk factors of bipolar disorder in the general population: A 
population-based cohort study. Bipolar Disorders, 15(3), 306313. doi: $10.1111 /$ bdi. 12058

Lieberman, D. Z., Massey, S. H., \& Goodwin, F. K. (2010). The role of gender in single vs married individuals with bipolar disorder. Comprehensive Psychiatry, 51(4), 380-385. doi: 10.1016/j.comppsych.2009.10.004

Meade, C. S., Fitzmaurice, G. M., Sanchez, A. K., Griffin, M. L., McDonald, L. J., \& Weiss, R. D. (2011). The relationship of manic episodes and drug abuse to sexual risk behavior in patients with co-occurring bipolar and substance use disorders: a 15-month prospective analysis. AIDS and Behavior, 15(8), 1829-33. doi: $10.1007 /$ s10461-010-9814-9

McElroy, S. L., Strakowski, S. M., Keck, P. E., Tugrul, K. L., West, S. A., \& Lonczak, H. S. (1995). Differences and similarities in mixed and pure mania. Comprehensive Psychiatry, 36(3), 187194. doi: $10.1016 / 0010-440 x(95) 90080-f$

Mohammad-Khani P, J., M., Jahani Tabesh, A., \& Tamanaie-far, S. (2013). [Structured clinical interview for DSM-IV-TR (Persian)]. Tehran: Danjeh Publication.

Olfson, M., Mojtabai, R., Merikangas, K., Compton, W., Wang S., Grant, B., et al. (2016). Reexamining associations between mania, depression, anxiety and substance use disorders: Results from a prospective national cohort. Molecular Psychiatry, 22(2), 235-241. doi: $10.1038 / \mathrm{mp} .2016 .64$

Proudfoot, J., Doran, J., Manicavasagar, V., \& Parker, G. (2011). The precipitants of manic/hypomanic episodes in the context of bipolar disorder: A review. Journal of Affective Disorders, 133(3), 381-387. doi: 10.1016/j.jad.2010.10.051

Sadock, B. J., \& Sadock, V. A. (2011). Kaplan and Sadock's synopsis of psychiatry: Behavioral sciences/clinical psychiatry. Philadelphia: Lippincott Williams \& Wilkins.

Sammarco, A. (2016). Women's health issues across the life cycle. Burlington: Jones \& Bartlett Publishers.

Schulze, T. G., Müller, D. J., Krauss, H., Gross, M., FangerauLefèvre, H., Illes, F., et al. (2002). Further evidence for age of onset being an indicator for severity in bipolar disorder. Journal of Affective Disorders, 68(2), 343-345. doi: 10.1016/s01650327(01)00306-8

Schwartzmann, Â. M., Amaral, J. A., Issler, C., Caetano, S. C., Tamada, R. S., Almeida, K. M. d., et al. (2007). A clinical study comparing manic and mixed episodes in patients with bipolar disorder. Revista Brasileira de Psiquiatria, 29(2), 130-133. doi: 10.1590/s1516-44462006005000036

Van der Voort, T. Y., Van Meijel, B., Hoogendoorn, A. W., Goossens, P. J., Beekman, A. T., \& Kupka, R. W. (2015). Collaborative care for patients with bipolar disorder: Effects on functioning and quality of life. Journal of Affective Disorders, 179, 14-22. doi: 10.1016/j.jad.2015.03.005

Yazla, E., İnanç, L., \& Bilici, M. (2012). [Relationship of sociodemographic features, clinical symptoms and functioning level among bipolar patients with manic episode and difference between these variables according to gender (Turkish)]. Dusunen Adam: The Journal of Psychiatry and Neurological Sciences, 25(3), 221. doi: 10.5350/dajpn2012250304

Young, R., Biggs, J., Ziegler, V., \& Meyer, D. (1978). A rating scale for mania: reliability, validity and sensitivity. The British Journal of Psychiatry, 133(5), 429-435. doi: 10.1192/bjp.133.5.429
Whitbourne, S. K., \& Halgin, R. (2014). Abnormal psychology: Clinical perspectives on psychological disorders with DSM-5 update. New York: McGraw-Hill Education. 
